\title{
Dietary intake of meat, fruits, vegetables, and selective micronutrients and risk of bladder cancer in the New England region of the United States
}

\author{
JW Wu', AJ Cross', D Baris', MH Ward', MR Karagas², A Johnson ${ }^{3}$, M Schwenn ${ }^{4}$, S Cherala $^{5}$, JS Colt', \\ KP Cantor ${ }^{1,6}$, N Rothman', DT Silverman' and R Sinha ${ }^{*, 1}$
}

'National Cancer Institute, National Institutes of Health, Department of Health and Human Services, 61 20 Executive Blvd, Rockville, MD 20852, USA; ${ }^{2}$ Dartmouth Medical School, Section of Biostatistics and Epidemiology, I Medical Center Drive, 7927 Rubin Building, Lebanon, NH 03756, USA; ${ }^{3}$ Vermont Cancer Registry, 108 Cherry Street, Burlington, VT 05402, USA; ${ }^{4}$ Maine Cancer Registry, 286 Water Street, Fourth Floor, II State House Station, Augusta, ME 04333, USA; ${ }^{5}$ New Hampshire department of Health and Human Services, I 29 Pleasant Street, Concord, NH 0330 I, USA;

${ }^{6}$ KP Cantor Environmental LLC, 708 Bonifant Street, Silver Spring, MD 20910, USA

\begin{abstract}
BACKGROUND: Despite many studies on diet and bladder cancer, there are areas that remain unexplored including meat mutagens, specific vegetable groups, and vitamins from diet.

METHODS: We conducted a population-based case-control study of bladder cancer in Maine, New Hampshire, and Vermont. A total of I I I I cases were ascertained through hospital pathology records and cancer registries from 200 I to 2004. Overall, I4I 8 controls were identified from the Department of Motor Vehicles ( $<65$ years) and Center for Medicaid and Medicare Services (65-79 years) and were frequency-matched to cases by state, sex, and age (within 5 years). Diet was assessed with a self-administered Diet History Questionnaire. Unconditional logistic regression was used to estimate odds ratios (OR) and 95\% confidence intervals (Cl).

RESULTS: Processed meat intake was positively associated with bladder cancer (highest vs lowest quartile OR: I.28; 95\% Cl: I.00-I.65; $\left.P_{\text {trend }}=0.035\right)$, with a stronger association for processed red meat $\left(\mathrm{OR}: 1.41 ; 95 \% \mathrm{Cl}: 1.08-1.84 ; P_{\text {trend }}=0.024\right)$. There were no associations between intake of fruits or vegetables and bladder cancer. We did, however, observe an inverse association with vitamin BI2 intake (OR: 0.77; 95\% Cl: 0.6I-0.99; $P=0.019$ ).

CONCLUSION: Vitamin BI 2 from diet may be protective against bladder cancer, whereas consuming processed meat may increase risk. British Journal of Cancer (2012) 106, I89|-1898. doi:I0.1038/bjc.2012.187 www.bjcancer.com
\end{abstract}

Published online 8 May 2012

(C) 2012 Cancer Research UK

Keywords: diet; micronutrients; bladder cancer

Over the past few decades, the incidence of bladder cancer has been relatively constant, with an estimated 70530 Americans (52 760 men and 17770 women) diagnosed with bladder cancer in 2010 (Howlader et al, 2010). Diet is a source of carcinogenic and anticarcinogenic compounds, which may be excreted through the urine (Pelucchi et al, 2006). However, there is a lack of consensus on the effects of diet on bladder cancer risk. In a 2007 review of the literature, no dietary components were 'convincingly', or even 'probably', associated with risk of bladder cancer (Marmot et al, 2007).

With the exception of one prospective cohort (Ferrucci et al, 2010) and one case-control study, (García-Closas et al, 2007) cooking preferences of meats, which determines the formation of mutagens such as heterocyclic amines (HCA) and polycyclic aromatic hydrocarbons (PAH), have not been studied extensively in relation to bladder cancer risk. However, these meat mutagens have been associated with an increased risk of breast, colorectal, upper gastrointestinal, lung, pancreatic, and prostate cancer (Alaejos et al, 2008; Zheng and Lee, 2009). Furthermore, HCAs

*Correspondence: Dr R Sinha; E-mail: sinhar@mail.nih.gov

Received I4 December 2011; revised 3 April 2012; accepted 7 April 2012; published online 8 May 2012 induce tumours in multiple organs in animal studies (Ohgaki et al, 1987), including the urinary tract (Hashida et al, 1982 Takahashi et al, 1993).

In general, prospective cohort and case-control studies have demonstrated inconsistent results for the association between fruits and vegetables and bladder cancer (Riboli et al, 1991; Shibata et al, 1992; Chyou et al, 1993; Bruemmer et al, 1996; Michaud et al, 1999; Nagano et al, 2000; Wakai et al, 2000; Balbi et al, 2001; Zeegers et al, 2001b; García-Closas et al, 2007). There is also a lack of consistent epidemiologic data that demonstrate one particular fruit or vegetable group is protective against bladder cancer, despite the fact that clinical studies that have shown specific vegetable groups, such as brassica and allium, activate glutathione S-transferases (GSTs), which act to minimise oxidative stress (Lampe et al, 2000; Asaduzzaman Khan et al, 2010). Furthermore, a recent meta-analysis has shown that deletion of GST M1 (GSTM1) may increase the risk of bladder cancer (Jiang et al, 2011).

Similarly, several prospective cohort and case-control studies have evaluated the role of micronutrients in bladder cancer with variable results (Steineck et al, 1990; Nomura et al, 1991; Riboli et al, 1991; Shibata et al, 1992; Bruemmer et al, 1996; Michaud et al, 1999; Michaud et al, 2000; Zeegers et al, 2001a; 
Michaud et al, 2002; García-Closas et al, 2007; Brinkman et al, 2010; Hotaling et al, 2011); however, there is evidence from laboratory and epidemiological studies that demonstrate certain micronutrients are associated with a reduced risk of specific cancer sites including the bladder (Greenwald et al, 2001; Leppert et al, 2006).

In the Spanish bladder cancer study (García-Closas et al, 2007), the authors found that higher compared with lower intakes of folate and B-vitamins were protective against bladder cancer and so our study aimed to confirm some of the results from that study. The Spanish bladder cancer study also analysed the role of meat cooking and related carcinogens. Therefore, we evaluated a range of dietary components, including meat (by cooking method and meat mutagens), fruits, vegetables, and micronutrients in relation to bladder cancer in a large population-based case-control study in northern New England.

\section{MATERIALS AND METHODS}

\section{Study population}

The details of the design of the New England Bladder Cancer Study have been published elsewhere (Baris et al, 2009). Briefly, newly diagnosed, histologically confirmed cases of urinary bladder carcinoma (including carcinoma in situ) aged 30-79 years were enroled in Maine, Vermont, and New Hampshire. Enrolment of cases occurred between 1 September 2001 and 31 October 2004 in Maine and Vermont and between 1 January 2002 and 31 July 2004 in New Hampshire. Cases were ascertained through hospital pathology departments, hospital cancer registries, and the state cancer registries. Out of 1878 patients who were eligible for study, $1213(65 \%)$ were interviewed. Reasons for non-participation included individuals who refused (50\%), were deceased $(22 \%)$, too ill (12\%), did not speak English fluently (5.5\%), had a physician refused (5\%), or could not be located (5\%). An expert pathologist conducted a blind review of the cases to confirm diagnosis, histological classification, and tumour stage and grade. After the review, 20 patients who were judged not to have bladder cancer and 22 who did not have urothelial carcinomas were excluded. We further excluded an additional 84 cases (7\%) because they did not complete the Diet History Questionnaire (DHQ) resulting in 1087 cases.

Controls aged 30-64 and 65-79 years were identified from Department of Motor Vehicles (DMV) records and by the Centers for Medicare and Medicaid Services (CMS), respectively. Controls had no previous history of bladder cancer and were frequency-matched to the bladder cancer cases by state, sex, and within 5 years of age at diagnosis of patients. There were 1418 control subjects interviewed, which accounted for $65 \%$ of the eligible individuals from the DMV and CMS (594 and 824, respectively). A total of 319 and 444 individuals from DMV and CMS, respectively, were eligible but did not participate, including refusals (DMV - 70\% and CMS - 65\%), those who could not be located (DMV - 24\% and CMS - 11\%), who did not speak English fluently (DMV - 3\% and CMS - 10\%), who were too ill (DMV - 1\% and CMS - 7\%), or deceased (DMV - $1 \%$ and CMS - 7\%). Similar to the cases, 125 controls (9\%) were not included in the analysis because the individuals did not complete the DHQ ensuing in 1293 control subjects.

Individuals who agreed and consented to the study were interviewed at home by a trained interviewer using a detailed computer-assisted personal interview. The interviewer obtained information on demographics, use of tobacco products, occupational and residential histories, fluid intake, use of hair-colouring products, family history of cancer, and medication use.

\section{Dietary assessment}

Food intake over the past 5 years was assessed with a modified version of the self-administered 124 food item DHQ created by the National Cancer Institute, which included portion size and dietary supplement questions (Subar et al, 2001). In general, we asked, 'During the last 5 years, how often did you eat...' in order to capture usual diet over the past 5 years. The DHQ was validated among an American population aged 50-71 years old from six states that did not include the New England region (Thompson et al, 2008); however, the New England study population is similar to the validation population such that they were generally older, White non-Hispanic participants. Correlations between the DHQ and $24 \mathrm{~h}$ dietary recalls for fruits and vegetables were 0.61 and 0.72 and for red meat was 0.70 and 0.62 for women and men, respectively (Thompson et al, 2008; Ferrucci et al, 2010).

The MyPyramid Equivalents Database (MPED 2.0) was utilised as a guideline to create food groups. Fruits were categorised as citrus/berries/melons (cantaloupe, melons, grapefruit, orange, and strawberry) and other fruits (apple, apricots, avocado, banana, grapes, peaches, nectarine, plum, pear, and plantain). Vegetables were grouped as cruciferous (coleslaw, cabbage/ sauerkraut, broccoli, and cauliflower/brussel sprouts), dark green (spinach (cooked and raw) and broccoli), orange (carrots and sweet potatoes), starchy (corn, peas, and potatoes), other vegetables (green beans, celery, olives, onions, lettuce, peppers, and tomatoes), and beans. Meat and fish were categorised as red (beef, veal, pork, and lamb), white (chicken and turkey), tuna, and other fish (fried and non-fried fish). Processed meat included red processed (ham, bacon, sausage, hot dog, and cold cuts) and white processed (turkey sausages and hot dogs, and poultry cold cuts). Vitamins from the diet were estimated by linking food codes from the United States Department of Agriculture's 1994 to 1996 Continuing Survey of Food Intakes by Individuals (CSFII) to those from the Nutrient Data Systems for Research (NDS-R) (Dixon et al, 2003).

Information was collected on how the meat and fish was cooked - baked, broiled, microwaved, pan-fried, or barbequed. Heterocyclic amines included 2-amino-3,4,8-trimethylimidazo (4,5-f)quinoxaline (DiMeIQx), 2-amino-3,8-dimethylimidazo(4,5-f) quinoxaline (MeIQx), and 2-amino-1-methyl-6-phenylimidazo (4,5-b)pyridine (PhIP) and benzo(a)pyrene $(\mathrm{B}(\mathrm{a}) \mathrm{P})$ intake was estimated as a marker for PAH. Total mutagenic activity, which measures mutagenicity from all meat-related mutagens, was also estimated (Ferrucci et al, 2010). Meat-cooking methods, HCAs, $\mathrm{B}(\mathrm{a}) \mathrm{P}$, and total mutagenic activity were analysed using Computerized Heterocylic Amines Resource for Research in Epidemiology of Disease (CHARRED) (Sinha et al, 2005).

\section{Statistical analysis}

Odds ratios (OR) and 95\% confidence intervals (CI) were estimated using unconditional logistic regression. For the meat variables, all models summed to total meat; for example, red and white meat were included in the same model, as was tuna and other fish. Dietary variables were energy-adjusted using the multivariate nutrient-density method. We excluded individuals with energy intake that fell in the 1st or 99th percentiles $(n=44)$. Quartiles were created for the dietary variables and determined based on the distribution among the controls. Multivariate models were adjusted for gender, age (0-54,55-64, 65-74, or $75+$ years), region (Maine, Vermont, New Hampshire), race (White or other), Hispanic status (yes or no), smoking status (never, occasional, former, or current), usual body mass index (BMI $-\mathrm{kg} \mathrm{m}^{-2}$, continuous), and total energy ( $\mathrm{kcal}$ per day, continuous). For the smoking status variable, 'never smokers' were defined as individuals who had $<100$ cigarettes in their lifetime. 'Occasional smokers' consumed $>100$ cigarettes but not on a regular basis 
(i.e. at least one cigarette per day for at least 6 months). 'Former smokers' were individuals who quit smoking 1 year or more before the diagnosis date for case patients or selection date for control subjects, whereas 'current smokers' were regularly smoking at the time of their interview or had quit $<1$ year before the reference date. More refined adjustment for smoking, including pack-years and number of cigarettes, for example, did not alter our results. Missing or unknown values were imputed with the median value of the variable. Individuals who did not have a known smoking status were excluded $(n=2)$. Adjustment for education, occupation, and use of non-steroidal anti-inflammatory drugs did not affect the association; therefore, these covariates were not included in the final model. $P$-values for linear trends were calculated using the quartile median values. Test for interactions were also conducted using the Wald statistic. Data analyses were calculated using SAS Version 9.1.3 (SAS Institute, Cary, NC, USA) and all $P$-values were two-tailed with an $\alpha$ level of 0.05 .

\section{RESULTS}

A total of 1068 cases (811 men and 257 women) and 1266 controls (927 men and 339 women) were included in our analysis (Table 1). The cases and controls were mostly from Maine and New Hampshire. The majority of the study subjects were White (94.5\% and $94.7 \%$ for cases and controls, respectively). Cases were more likely to be current smokers compared with controls $(30.1 \%$ vs $13.5 \%, P<0.001)$. BMI was similar among cases $\left(26.2 \pm 4.4 \mathrm{~kg} \mathrm{~m}^{-2}\right)$ and controls $\left(26.0 \pm 4.1 \mathrm{~kg} \mathrm{~m}^{-2}\right)$. Total energy intake among cases and controls was $2041 \pm 793$ and

Table I Characteristics of cases and controls ${ }^{\mathrm{a}}$

\begin{tabular}{|c|c|c|c|}
\hline Variable & Cases & Controls & $P_{\text {value }}{ }^{c}$ \\
\hline$N(\%)$ & I068 (45.8) & $1266(54.2)$ & \\
\hline $\begin{array}{l}\text { Region } \\
\text { Maine } \\
\text { Vermont } \\
\text { New Hampshire }\end{array}$ & $\begin{array}{l}523(49.0) \\
188(17.6) \\
357(33.4)\end{array}$ & $\begin{array}{l}664(52.5) \\
226(17.8) \\
376(29.7)\end{array}$ & 0.138 \\
\hline $\begin{array}{l}\text { Age (years) } \\
\text { 30-54 } \\
55-64 \\
65-74 \\
75+\end{array}$ & $\begin{array}{l}158(14.8) \\
274(25.7) \\
404(37.8) \\
232(21.7)\end{array}$ & $\begin{array}{l}195(15.4) \\
305(24.1) \\
504(39.8) \\
262(20.7)\end{array}$ & 0.662 \\
\hline $\begin{array}{l}\text { Gender } \\
\text { Male } \\
\text { Female }\end{array}$ & $\begin{array}{l}811(75.0) \\
257(24.1)\end{array}$ & $\begin{array}{l}927(73.2) \\
339(26.8)\end{array}$ & 0.134 \\
\hline $\begin{array}{l}\text { Race } \\
\text { White } \\
\text { Native Americans } \\
\text { Other }\end{array}$ & $\begin{array}{r}1009(94.5) \\
52(4.9) \\
7(0.7)\end{array}$ & $\begin{array}{r}1197(94.7) \\
53(4.2) \\
14(1.1)\end{array}$ & 0.386 \\
\hline $\begin{array}{l}\text { Hispanic status } \\
\text { Yes } \\
\text { No }\end{array}$ & $\begin{array}{r}20(1.9) \\
1048(98.1)\end{array}$ & $\begin{array}{c}20(1.6) \\
1246(98.4)\end{array}$ & 0.587 \\
\hline $\begin{array}{l}\text { Smoking status } \\
\text { Non-smoker } \\
\text { Occasional } \\
\text { Former } \\
\text { Current }\end{array}$ & $\begin{aligned} 162 & (15.2) \\
18 & (1.7) \\
567 & (53.1) \\
321 & (30.1)\end{aligned}$ & $\begin{aligned} 425 & (33.6) \\
39 & (3.1) \\
631 & (49.8) \\
171 & (13.5)\end{aligned}$ & $<0.001$ \\
\hline $\begin{array}{l}\text { Usual BMI }\left(\mathrm{kgm}^{-2}\right)^{\mathrm{b}} \\
\text { Energy (kcal per day) }\end{array}$ & $\begin{array}{c}26.2 \pm 4.4 \\
2041 \pm 793\end{array}$ & $\begin{array}{c}26.0 \pm 4.1 \\
|987 \pm 71|\end{array}$ & $\begin{array}{l}0.189 \\
0.081\end{array}$ \\
\hline
\end{tabular}

Abbreviation: $\mathrm{BMI}=$ body mass index. ${ }^{\mathrm{a}}$ The numbers in brackets are percentages. ${ }^{b}$ This is a continuous variable and a mean and a SD are given in the format of mean $\pm \mathrm{SD}$. ${ }^{\mathrm{C}} \mathrm{P}$-values were tabulated using $\chi^{2}$ tests (categorical variables) or $t$-tests (continuous variables).
$1987 \pm 711 \mathrm{kcal}$ per day, respectively. A comparison between the controls recruited from the DMV vs CMS showed no difference in the baseline characteristics except for region and total energy intake; a higher proportion of controls from the DMV were from Vermont compared with CMS controls, and controls from the DMV had a higher total energy intake compared with those from CMS (2089 \pm 707 and $1920 \pm 706 \mathrm{kcal}$, respectively).

Individuals in the highest, compared with those in the lowest, quartile of processed meat intake had an increased risk of bladder cancer in multivariate models (OR: 1.28; 95\% CI: 1.00-1.65; $P_{\text {trend }}=0.035$; Table 2); the association was slightly stronger for processed red meat specifically (OR: 1.41 ; 95\% CI: 1.08-1.84; $\left.P_{\text {trend }}=0.024\right)$. In contrast, no association with bladder cancer was

Table 2 Meat and fish intake and risk of bladder cancer ${ }^{\mathrm{a}, \mathrm{b}}$

\begin{tabular}{|c|c|c|c|c|c|}
\hline \multirow{2}{*}{$\begin{array}{l}\text { Quartiles } \\
\text { of intake }\end{array}$} & \multirow{2}{*}{$\begin{array}{c}\text { Median } \\
(\mathrm{g} \text { per } 1000 \mathrm{kcal})\end{array}$} & \multirow[b]{2}{*}{ Cases } & \multirow[b]{2}{*}{ Controls } & \multicolumn{2}{|c|}{ Multivariate model ${ }^{c}$} \\
\hline & & & & OR & $95 \% \mathrm{Cl}$ \\
\hline $\begin{array}{l}\text { Red meat } \\
\text { Q1 } \\
\text { Q2 } \\
\text { Q3 } \\
\text { Q4 } \\
P_{\text {trend }}\end{array}$ & $\begin{array}{l}17.2 \\
27.6 \\
37.4 \\
53.0\end{array}$ & $\begin{array}{l}241 \\
254 \\
269 \\
304\end{array}$ & $\begin{array}{l}316 \\
317 \\
317 \\
316\end{array}$ & $\begin{array}{l}1.00 \\
0.97 \\
1.04 \\
1.14 \\
0.258\end{array}$ & $\begin{array}{l}(0.76,1.24) \\
(0.81,1.33) \\
(0.89,1.46)\end{array}$ \\
\hline $\begin{array}{l}\text { White meat } \\
\text { Q1 } \\
\text { Q2 } \\
\text { Q3 } \\
\text { Q4 } \\
P_{\text {trend }}\end{array}$ & $\begin{array}{r}9.6 \\
17.6 \\
27.7 \\
51.0\end{array}$ & $\begin{array}{l}320 \\
267 \\
236 \\
245\end{array}$ & $\begin{array}{l}316 \\
317 \\
316 \\
317\end{array}$ & $\begin{array}{l}1.00 \\
0.94 \\
0.85 \\
0.89 \\
0.428\end{array}$ & $\begin{array}{l}(0.73,1.20) \\
(0.65,1.11) \\
(0.67,1.18)\end{array}$ \\
\hline $\begin{array}{l}\text { Processed me } \\
\text { Q1 } \\
\text { Q2 } \\
\text { Q3 } \\
\text { Q4 } \\
P_{\text {trend }}\end{array}$ & $\begin{array}{r}2.9 \\
6.1 \\
10.1 \\
18.4\end{array}$ & $\begin{array}{l}226 \\
246 \\
287 \\
309\end{array}$ & $\begin{array}{l}316 \\
317 \\
316 \\
317\end{array}$ & $\begin{array}{l}1.00 \\
1.01 \\
1.19 \\
1.28 \\
0.035\end{array}$ & $\begin{array}{l}(0.78,1.30) \\
(0.92,1.53) \\
(1.00,1.65)\end{array}$ \\
\hline $\begin{array}{l}\text { Red, proce } \\
\text { Q1 } \\
\text { Q2 } \\
\text { Q3 } \\
\text { Q4 } \\
P_{\text {trend }}\end{array}$ & $\begin{array}{r}1.9 \\
4.3 \\
7.4 \\
13.5\end{array}$ & $\begin{array}{l}200 \\
263 \\
289 \\
316\end{array}$ & $\begin{array}{l}316 \\
317 \\
316 \\
317\end{array}$ & $\begin{array}{l}1.00 \\
1.24 \\
1.39 \\
1.41 \\
0.024\end{array}$ & $\begin{array}{l}(0.96,1.60) \\
(1.07,1.81) \\
(1.08,1.84)\end{array}$ \\
\hline $\begin{array}{l}\text { White, pr } \\
\text { Q1 } \\
\text { Q2 } \\
\text { Q3 } \\
\text { Q4 } \\
P_{\text {trend }}\end{array}$ & essed & $\begin{array}{l}278 \\
243 \\
270 \\
277\end{array}$ & $\begin{array}{l}317 \\
316 \\
317 \\
316\end{array}$ & $\begin{array}{l}1.00 \\
0.99 \\
1.07 \\
1.17 \\
0.192\end{array}$ & $\begin{array}{l}(0.77,1.26) \\
(0.84,1.36) \\
(0.91,1.49)\end{array}$ \\
\hline $\begin{array}{l}\text { Total tuna } \\
\text { Q1 } \\
\text { Q2 } \\
\text { Q3 } \\
\text { Q4 } \\
\text { P trend }^{\text {ton }}\end{array}$ & $\begin{array}{l}0.3 \\
1.1 \\
2.2 \\
5.3\end{array}$ & $\begin{array}{l}262 \\
254 \\
295 \\
257\end{array}$ & $\begin{array}{l}316 \\
317 \\
317 \\
316\end{array}$ & $\begin{array}{l}1.00 \\
1.11 \\
1.23 \\
1.10 \\
0.975\end{array}$ & $\begin{array}{l}(0.87,1.43) \\
(0.96,1.58) \\
(0.85,1.43)\end{array}$ \\
\hline $\begin{array}{l}\text { Other fish } \\
\text { Q1 } \\
\text { Q2 } \\
\text { Q3 } \\
\text { Q4 } \\
P_{\text {trend }}\end{array}$ & $\begin{array}{l}0.8 \\
2.2 \\
4.1 \\
8.9\end{array}$ & $\begin{array}{l}356 \\
224 \\
235 \\
253\end{array}$ & $\begin{array}{l}316 \\
317 \\
317 \\
316\end{array}$ & $\begin{array}{l}1.00 \\
0.65 \\
0.74 \\
0.84 \\
0.452\end{array}$ & $\begin{array}{l}(0.51,0.83) \\
(0.57,0.94) \\
(0.65,1.10)\end{array}$ \\
\hline
\end{tabular}

Abbreviations: $\mathrm{Cl}=$ confidence intervals; $\mathrm{OR}=$ odds ratios. ${ }^{\mathrm{a}} P_{\text {trends }}$ were calculated using the median value for each quartile. ${ }^{\mathrm{b}}$ All models included total meat and fish intakes. 'Adjusted for gender (female/male), age (0-54, 55-64, 65-74, $75+$ years), region (Maine, Vermont, New Hampshire), race (White/other), Hispanic status (yes/no), smoking status (never, occasional, former, current), usual BMI $\left(\mathrm{kg} \mathrm{m}^{-2}-\right.$ continuous), and total energy (kcal per day - continuous). 
Table 3 Meat-cooking methods and risk of bladder cancer ${ }^{\mathrm{a}}$

\begin{tabular}{|c|c|c|c|c|c|}
\hline \multirow{2}{*}{$\begin{array}{l}\text { Quartiles } \\
\text { of intake }\end{array}$} & \multirow{2}{*}{$\begin{array}{c}\text { Median } \\
\text { (g per } 1000 \mathrm{kcal})\end{array}$} & \multirow[b]{2}{*}{ Cases } & \multirow[b]{2}{*}{ Controls } & \multicolumn{2}{|c|}{ Multivariate model } \\
\hline & & & & OR & $95 \% \mathrm{Cl}$ \\
\hline \multicolumn{6}{|c|}{ Baking/microwaving/broiling } \\
\hline QI & 0.4 & 261 & 317 & 1.00 & \\
\hline Q2 & 2.2 & 270 & 316 & 1.07 & $(0.84,1.36)$ \\
\hline Q3 & 5.0 & 286 & 317 & 1.13 & $(0.89,1.44)$ \\
\hline Q4 & 12.6 & 251 & 316 & 1.00 & $(0.78,1.29)$ \\
\hline$P_{\text {trend }}$ & & & & 0.814 & \\
\hline \multicolumn{6}{|l|}{ Barbequed } \\
\hline QI & 0 & 260 & 317 & 1.00 & \\
\hline Q2 & 1.5 & 298 & 316 & 1.15 & $(0.91,1.47)$ \\
\hline Q3 & 4.1 & 265 & 316 & 1.04 & $(0.81,1.32)$ \\
\hline Q4 & 10.2 & 245 & 317 & 1.00 & $(0.78,1.29)$ \\
\hline$P_{\text {trend }}$ & & & & 0.649 & \\
\hline \multicolumn{6}{|l|}{ Pan-fried } \\
\hline Q। & 0.2 & 228 & 317 & 1.00 & \\
\hline Q2 & 1.6 & 290 & 316 & 1.23 & $(0.96,1.57)$ \\
\hline Q3 & 3.9 & 239 & 316 & 0.92 & $(0.71,1.18)$ \\
\hline Q4 & 9.5 & 311 & 317 & 1.10 & $(0.86,1.40)$ \\
\hline$P_{\text {trend }}$ & & & & 0.942 & \\
\hline
\end{tabular}

Abbreviations: $\mathrm{Cl}=$ confidence intervals; $\mathrm{OR}=$ odds ratios. ${ }^{\mathrm{a}} P_{\text {trends }}$ were calculated using the median value for each quartile. ${ }^{b}$ Adjusted for gender (female/male), age (0-54, 55-64, 65-74, 75 + years), region (Maine, Vermont, New Hampshire), race (White/other), Hispanic status (yes/no), smoking status (never, occasional, former, current), usual BMI ( $\mathrm{kgm}^{-2}$ - continuous), and total energy (kcal per day continuous).

apparent for intake of processed white meat (high $v s$ low intake: OR: $1.17 ; 95 \%$ CI: $0.91-1.49 ; P_{\text {trend }}=0.192$ ). Given that a positive association for processed red meat was observed, we investigated specific processed meat groups. There was no significant linear, increased risk of bladder cancer associated with intake of bacon, or sausages; however, those in the highest intake category of ham (not lunchmeat), cold cuts, and hot dog compared with the lowest had a moderate, non-significant increase in risk (OR: 1.29; 95\% CI: $1.00-1.68 ; P_{\text {trend }}=0.072$; OR: $1.21 ; 95 \%$ CI: $0.94-1.55$; $P_{\text {trend }}=0.047$; and OR: $1.18 ; 95 \%$ CI: $0.91-1.53 ; P_{\text {trend }}=0.026$, respectively). We did not observe any association between consumption of tuna or other types of fish and bladder cancer.

Barbequed and pan-fried meats were not associated with bladder cancer when comparing those in the highest with those in the lowest quartile (OR: $1.00 ; 95 \%$ CI: $0.78-1.29 ; P_{\text {trend }}=0.649$ and OR: $1.10 ; 95 \%$ CI: $0.86-1.40 ; P_{\text {trend }}=0.942$; respectively) (Table 3). Furthermore, there was no association between intakes of MeIQx, DiMeIQx, PhIP, $\mathrm{B}(\alpha) \mathrm{P}$, total mutagenicity, or haeme iron intake and bladder cancer (Table 4).

Although the unadjusted models indicated inverse associations between the 'citrus, melons and berries' food group, as well as 'cruciferous vegetables', 'dark green vegetables' and 'orange vegetables' and bladder cancer (data not shown), these associations were attenuated after multivariate adjustment (Table 5). The smoking variable was the strongest confounder for the association between the fruit and vegetable groups and bladder cancer.

In general, vitamins and minerals obtained from the diet were not associated with bladder cancer after multivariate adjustment (Table 6), with the exception of vitamin B12. Individuals in the highest, compared with the lowest, quartile of vitamin B12 intake had a lower risk of bladder cancer (OR: 0.77; 95\% CI: 0.61-0.99; $P=0.019)$. Vitamins acquired from supplements were not associated with bladder cancer, including vitamin B12 (data not shown).

For most dietary variables, we did not find any differences by smoking status, except among never smokers we observed decreased risks for those in the highest $v s$ lowest category of vegetables, vitamin $\mathrm{E}$, and $\alpha$-carotene (Supplementary Table 1), but none
Table 4 Meat mutagens and haeme iron in relation to bladder cancer ${ }^{2}$

\begin{tabular}{|c|c|c|c|c|c|}
\hline \multirow{2}{*}{$\begin{array}{l}\text { Quartiles } \\
\text { of intake }\end{array}$} & \multirow[b]{2}{*}{ Median } & \multirow[b]{2}{*}{ Cases } & \multirow[b]{2}{*}{ Controls } & \multicolumn{2}{|c|}{ Multivariate model $^{\mathrm{b}}$} \\
\hline & & & & OR & $95 \% \mathrm{Cl}$ \\
\hline \multicolumn{6}{|l|}{ MelQx (ngs) } \\
\hline QI & 2.9 & 243 & 317 & 1.00 & \\
\hline Q2 & 8.4 & 265 & 316 & 1.10 & $(0.86,1.4 I)$ \\
\hline Q3 & 17.6 & 252 & 317 & 0.95 & $(0.74,1.22)$ \\
\hline Q4 & 43.7 & 308 & 316 & 1.04 & $(0.81,1.33)$ \\
\hline$P_{\text {trend }}$ & & & & 0.999 & \\
\hline \multicolumn{6}{|c|}{ DiMelQx (ngs) } \\
\hline QI & 0 & 245 & 317 & 1.00 & \\
\hline Q2 & 0.4 & 274 & 317 & 1.10 & $(0.86,1.40)$ \\
\hline Q3 & 1.0 & 265 & 316 & 1.02 & $(0.80,1.30)$ \\
\hline Q4 & 3.0 & 284 & 316 & 1.01 & $(0.79,1.30)$ \\
\hline$P_{\text {trend }}$ & & & & 0.822 & \\
\hline \multicolumn{6}{|l|}{ PhIP (ngs) } \\
\hline QI & 5.2 & 250 & 317 & 1.00 & \\
\hline Q2 & 17.2 & 246 & 316 & 0.95 & $(0.74,1.21)$ \\
\hline Q3 & 39.1 & 329 & 317 & 1.26 & $(0.99,1.60)$ \\
\hline Q4 & 117.6 & 243 & 316 & 0.90 & $(0.70,1.17)$ \\
\hline$P_{\text {trend }}$ & & & & 0.334 & \\
\hline \multicolumn{6}{|l|}{$B(a) P$ (ngs) } \\
\hline QI & 0.3 & 264 & 317 & 1.00 & \\
\hline Q2 & 1.8 & 278 & 316 & 1.06 & $(0.83,1.34)$ \\
\hline Q3 & 8.9 & 266 & 317 & 1.01 & $(0.79,1.28)$ \\
\hline Q4 & 39.7 & 260 & 316 & 1.02 & $(0.80,1.31)$ \\
\hline$P_{\text {trend }}$ & & & & 0.969 & \\
\hline \multicolumn{6}{|c|}{ Mutagenic activity } \\
\hline QI & 430.1 & 258 & 317 & 1.00 & \\
\hline Q2 & 1087.7 & 243 & 316 & 0.92 & $(0.72,1.18)$ \\
\hline Q3 & 2166.0 & 291 & 317 & 1.04 & $(0.82,1.33)$ \\
\hline Q4 & 5415.2 & 276 & 316 & 0.93 & $(0.72,1.19)$ \\
\hline$P_{\text {trend }}$ & & & & 0.652 & \\
\hline \multicolumn{6}{|c|}{ Haeme iron (mcg) } \\
\hline QI & 111.6 & 243 & 317 & 1.00 & \\
\hline Q2 & 220.5 & 257 & 316 & 1.01 & $(0.78,1.28)$ \\
\hline Q3 & 338.1 & 262 & 317 & 0.99 & $(0.77,1.28)$ \\
\hline Q4 & 565.5 & 306 & 316 & 1.08 & $(0.82,1.42)$ \\
\hline$P_{\text {trend }}$ & & & & 0.558 & \\
\hline
\end{tabular}

Abbreviations: $\mathrm{Cl}=$ confidence intervals; $\mathrm{OR}=$ odds ratios. ${ }^{\mathrm{a}} \mathrm{P}_{\text {trends }}$ were calculated using the median value for each quartile. ${ }^{b}$ Adjusted for gender (female/male), age (0-54, 55-64, 65-74, 75 + years), region (Maine, Vermont, New Hampshire), race (White/other), Hispanic status (yes/no), smoking status (never, occasional, former, current), usual BMI $\left(\mathrm{kgm}^{-2}\right.$ - continuous), and total energy (kcal per day continuous).

of the interaction terms were statistically significant $(P>0.05)$. Other results stratified by age, gender, region, or BMI were not materially different from the overall findings (data not shown).

\section{DISCUSSION}

In this large population-based case-control study, higher intake of processed meat, and processed red meat in particular, was associated with an increased risk of bladder cancer; although meat-cooking methods, HCAs and $\mathrm{B}(\mathrm{a}) \mathrm{P}$ were not associated with risk. We found no statistically significant associations between fruits or vegetables and bladder cancer. Individuals in the highest quartile of dietary vitamin B12 intake had a decreased risk of bladder cancer.

In agreement with our findings, many previous studies have not found an association between total or red meat and bladder cancer (Riboli et al, 1991; Chyou et al, 1993; Tavani et al, 2000; Wakai et al, 2000; Balbi et al, 2001; Cross et al, 2007; 
Table 5 Fruit and vegetable intake and risk of bladder cancer ${ }^{a}$

\begin{tabular}{|c|c|c|c|c|c|}
\hline \multirow{2}{*}{$\begin{array}{l}\text { Quartiles } \\
\text { of intake }\end{array}$} & \multirow{2}{*}{$\begin{array}{c}\text { Median } \\
\text { (g per } 1000 \mathrm{kcal})\end{array}$} & \multirow[b]{2}{*}{ Cases } & \multirow[b]{2}{*}{ Controls } & \multicolumn{2}{|c|}{ Multivariate model } \\
\hline & & & & OR & $95 \% \mathrm{Cl}$ \\
\hline $\begin{array}{l}\text { Total Fruits } \\
\text { Q1 } \\
\text { Q2 } \\
\text { Q3 } \\
\text { Q4 } \\
P_{\text {trend }}\end{array}$ & $\begin{array}{r}33.6 \\
83.0 \\
135.4 \\
227.7\end{array}$ & $\begin{array}{l}295 \\
267 \\
256 \\
250\end{array}$ & $\begin{array}{l}317 \\
316 \\
316 \\
317\end{array}$ & $\begin{array}{l}1.00 \\
1.11 \\
1.11 \\
1.13 \\
0.404\end{array}$ & $\begin{array}{l}(0.87,1.42) \\
(0.86,1.42) \\
(0.88,1.46)\end{array}$ \\
\hline $\begin{array}{l}\text { Citrus, me } \\
\text { Q1 } \\
\text { Q2 } \\
\text { Q3 } \\
\text { Q4 } \\
P_{\text {trend }}\end{array}$ & $\begin{array}{c}\text { elons, and berries } \\
3.9 \\
8.6 \\
15.5 \\
38.5\end{array}$ & $\begin{array}{l}298 \\
281 \\
262 \\
227\end{array}$ & $\begin{array}{l}317 \\
316 \\
317 \\
316\end{array}$ & $\begin{array}{l}1.00 \\
1.06 \\
1.03 \\
0.94 \\
0.444\end{array}$ & $\begin{array}{l}(0.84,1.35) \\
(0.81,1.31) \\
(0.73,1.21)\end{array}$ \\
\hline $\begin{array}{l}\text { Other fru } \\
\text { Q1 } \\
\text { Q2 } \\
\text { Q3 } \\
\text { Q4 } \\
P_{\text {trend }}\end{array}$ & $\begin{array}{r}7.9 \\
24.4 \\
49.3 \\
94.6\end{array}$ & $\begin{array}{l}304 \\
259 \\
262 \\
243\end{array}$ & $\begin{array}{l}316 \\
317 \\
317 \\
316\end{array}$ & $\begin{array}{l}1.00 \\
1.01 \\
1.16 \\
1.08 \\
0.447\end{array}$ & $\begin{array}{l}(0.79,1.27) \\
(0.91,1.48) \\
(0.84,1.39)\end{array}$ \\
\hline $\begin{array}{l}\text { Total vegetal } \\
\text { Q1 } \\
\text { Q2 } \\
\text { Q3 } \\
\text { Q4 } \\
P_{\text {trend }}\end{array}$ & $\begin{array}{r}64.6 \\
99.1 \\
130.3 \\
187.8\end{array}$ & $\begin{array}{l}290 \\
261 \\
282 \\
235\end{array}$ & $\begin{array}{l}316 \\
317 \\
316 \\
317\end{array}$ & $\begin{array}{l}1.00 \\
0.97 \\
1.06 \\
0.90 \\
0.495\end{array}$ & $\begin{array}{l}(0.76,1.23) \\
(0.84,1.35) \\
(0.70,1.16)\end{array}$ \\
\hline $\begin{array}{c}\text { Cruciferol } \\
\text { Q1 } \\
\text { Q2 } \\
\text { Q3 } \\
\text { Q4 } \\
P_{\text {trend }}\end{array}$ & $\begin{array}{r}\text { us vegetables } \\
2.6 \\
7.6 \\
13.4 \\
23.3\end{array}$ & $\begin{array}{l}305 \\
264 \\
274 \\
225\end{array}$ & $\begin{array}{l}316 \\
317 \\
316 \\
317\end{array}$ & $\begin{array}{l}1.00 \\
0.93 \\
0.99 \\
0.84 \\
0.192\end{array}$ & $\begin{array}{l}(0.74,1.18) \\
(0.78,1.25) \\
(0.65,1.07)\end{array}$ \\
\hline $\begin{array}{c}\text { Dark gree } \\
\text { Q1 } \\
\text { Q2 } \\
\text { Q3 } \\
\text { Q4 } \\
P_{\text {trend }}\end{array}$ & $\begin{array}{r}\text { en vegetables } \\
1.2 \\
4.1 \\
9.0 \\
22.7\end{array}$ & $\begin{array}{l}325 \\
248 \\
265 \\
230\end{array}$ & $\begin{array}{l}317 \\
316 \\
316 \\
317\end{array}$ & $\begin{array}{l}1.00 \\
0.86 \\
0.89 \\
0.82 \\
0.199\end{array}$ & $\begin{array}{l}(0.68,1.09) \\
(0.70,1.13) \\
(0.64,1.05)\end{array}$ \\
\hline $\begin{array}{c}\text { Orange v } \\
\text { Q1 } \\
\text { Q2 } \\
\text { Q3 } \\
\text { Q4 } \\
P_{\text {trend }}\end{array}$ & $\begin{array}{l}\text { egetables } \\
0.7 \\
1.8 \\
3.5 \\
8.3\end{array}$ & $\begin{array}{l}329 \\
268 \\
251 \\
220\end{array}$ & $\begin{array}{l}316 \\
317 \\
317 \\
316\end{array}$ & $\begin{array}{l}1.00 \\
0.92 \\
0.90 \\
0.82 \\
0.150\end{array}$ & $\begin{array}{l}(0.72,1.16) \\
(0.71,1.14) \\
(0.64,1.06)\end{array}$ \\
\hline $\begin{array}{c}\text { Starchy ve } \\
\text { Q1 } \\
\text { Q2 } \\
\text { Q3 } \\
\text { Q4 } \\
P_{\text {trend }}\end{array}$ & $\begin{array}{r}\text { egetables } \\
\qquad \begin{array}{r}14.4 \\
27.5 \\
41.4 \\
63.9\end{array}\end{array}$ & $\begin{array}{l}264 \\
275 \\
272 \\
247\end{array}$ & $\begin{array}{l}316 \\
317 \\
317 \\
316\end{array}$ & $\begin{array}{l}1.00 \\
0.98 \\
1.03 \\
0.93 \\
0.662\end{array}$ & $\begin{array}{l}(0.77,1.24) \\
(0.81,1.31) \\
(0.73,1.20)\end{array}$ \\
\hline $\begin{array}{l}\text { Other ves } \\
\text { Q1 } \\
\text { Q2 } \\
\text { Q3 } \\
\text { Q4 } \\
P_{\text {trend }}\end{array}$ & $\begin{array}{r}\text { getables } \\
\qquad \begin{array}{r}14.9 \\
26.6 \\
39.7 \\
66.3\end{array}\end{array}$ & $\begin{array}{l}283 \\
267 \\
251 \\
267\end{array}$ & $\begin{array}{l}317 \\
316 \\
317 \\
316\end{array}$ & $\begin{array}{l}1.00 \\
1.02 \\
0.99 \\
1.03 \\
0.822\end{array}$ & $\begin{array}{l}(0.81,1.31) \\
(0.78,1.26) \\
(0.81,1.32)\end{array}$ \\
\hline $\begin{array}{l}\text { Total Beans } \\
\text { Q1 } \\
\text { Q2 } \\
\text { Q3 } \\
\text { Q4 } \\
P_{\text {trend }}\end{array}$ & $\begin{array}{r}0.6 \\
2.5 \\
5.6 \\
12.1\end{array}$ & $\begin{array}{l}302 \\
269 \\
241 \\
256\end{array}$ & $\begin{array}{l}316 \\
317 \\
317 \\
316\end{array}$ & $\begin{array}{l}1.00 \\
0.97 \\
0.88 \\
0.95 \\
0.686\end{array}$ & $\begin{array}{l}(0.77,1.24) \\
(0.69,1.12) \\
(0.75,1.22)\end{array}$ \\
\hline
\end{tabular}

Abbreviations: $\mathrm{Cl}=$ confidence intervals; $\mathrm{OR}=$ odds ratios. ${ }^{\mathrm{a}}{ }_{\text {trends }}$ were calculated using the median value for each quartile. ${ }^{b}$ Adjusted for gender (female/male), age (0-54, 55-64, 65-74, 75 + years), region (Maine, Vermont, New Hampshire), race (White/ other), Hispanic status (yes/no), smoking status (never, occasional, former, current), usual BMI ( $\mathrm{kg} \mathrm{m}^{-2}$ - continuous), and total energy (kcal per day - continuous).
Table 6 Vitamins from diet and risk of bladder cancer ${ }^{\mathrm{a}}$

\begin{tabular}{|c|c|c|c|c|c|}
\hline \multirow{2}{*}{$\begin{array}{l}\text { Quartiles } \\
\text { of intake }\end{array}$} & \multirow[b]{2}{*}{ Median } & \multirow[b]{2}{*}{ Cases } & \multirow[b]{2}{*}{ Controls } & \multicolumn{2}{|c|}{ Multivariate model ${ }^{b}$} \\
\hline & & & & OR & $95 \% \mathrm{Cl}$ \\
\hline \multicolumn{6}{|c|}{ Vitamin A (mcg per $1000 \mathrm{kcal})$} \\
\hline QI & 279.8 & 307 & 316 & 1.00 & \\
\hline Q2 & 356.4 & 276 & 317 & 0.98 & $(0.78,1.24)$ \\
\hline Q3 & 432.9 & 257 & 317 & 0.94 & $(0.74,1.20)$ \\
\hline Q4 & 551.3 & 228 & 316 & 0.85 & $(0.66,1.08)$ \\
\hline$P_{\text {trend }}$ & & & & 0.165 & \\
\hline
\end{tabular}

Vitamin B2 (mg per $1000 \mathrm{kcal})$

$\begin{array}{llllll}\text { Q1 } & 0.7 & 293 & 317 & 1.00 & \\ \text { Q2 } & 0.9 & 289 & 316 & 1.05 & (0.83,1.33) \\ \text { Q3 } & 1.0 & 265 & 317 & 1.00 & (0.79,1.27) \\ \text { Q4 } & 1.3 & 221 & 316 & 0.90 & (0.70,1.15) \\ P_{\text {trend }} & & & & 0.308 & \end{array}$

Vitamin B6 (mg per $1000 \mathrm{kcal})$

$\begin{array}{llllll}\text { Q1 } & 0.7 & 307 & 317 & 1.00 & \\ \text { Q2 } & 0.9 & 295 & 316 & 1.11 & (0.88,1.41) \\ \text { Q3 } & 1.1 & 241 & 316 & 0.96 & (0.75,1.22) \\ \text { Q4 } & 1.4 & 225 & 317 & 0.93 & (0.72,1.20) \\ P_{\text {trend }} & & & & 0.372 & \end{array}$

Vitamin B/2 (mcg per $1000 \mathrm{kcal})$

$\begin{array}{llllll}\text { Q1 } & 1.8 & 315 & 316 & 1.00 & \\ \text { Q2 } & 2.4 & 282 & 317 & 0.92 & (0.73,1.17) \\ \text { Q3 } & 3.1 & 233 & 317 & 0.76 & (0.60,0.97) \\ \text { Q4 } & 4.1 & 238 & 316 & 0.77 & (0.61,0.99) \\ P_{\text {trend }} & & & & 0.019 & \end{array}$

Vitamin C (mg per $1000 \mathrm{kcal})$

$\begin{array}{llllll}\text { Q1 } & 32.3 & 288 & 317 & 1.00 & \\ \text { Q2 } & 53.1 & 261 & 316 & 1.06 & (0.83,1.35) \\ \text { Q3 } & 71.6 & 257 & 316 & 1.10 & (0.86,1.40) \\ \text { Q4 } & 104.6 & 262 & 317 & 1.15 & (0.90,1.47) \\ P_{\text {trend }} & & & & 0.265 & \end{array}$

Vitamin $E(\mathrm{mg}$ per $1000 \mathrm{kcal})$

$\begin{array}{llllll}\text { Q1 } & 2.9 & 335 & 317 & 1.00 & \\ \text { Q2 } & 3.6 & 266 & 316 & 0.91 & (0.72,1.15) \\ \text { Q3 } & 4.2 & 223 & 317 & 0.79 & (0.62,1.00) \\ \text { Q4 } & 5.6 & 244 & 316 & 0.91 & (0.71,1.15) \\ P_{\text {trend }} & & & & 0.405 & \end{array}$

Folate (mcg per $1000 \mathrm{kcal})$

$\begin{array}{llllll}\text { Q1 } & 138.8 & 286 & 317 & 1.00 & \\ \text { Q2 } & 174.4 & 286 & 316 & 1.10 & (0.87,1.40) \\ \text { Q3 } & 205.7 & 259 & 317 & 1.10 & (0.86,1.41) \\ \text { Q4 } & 255.8 & 237 & 316 & 1.06 & (0.82,1.37) \\ P_{\text {trend }} & & & & 0.708 & \end{array}$

$\alpha$-Carotene (mcg per $1000 \mathrm{kcal})$

$\begin{array}{lrrlll}\text { Q1 } & 99.3 & 283 & 316 & 1.00 & \\ \text { Q2 } & 171.9 & 282 & 317 & 1.08 & (0.85,1.37) \\ \text { Q3 } & 262.2 & 277 & 316 & 1.06 & (0.84,1.35) \\ \text { Q4 } & 468.1 & 226 & 317 & 0.94 & (0.73,1.20) \\ P_{\text {trend }} & & & & 0.449 & \end{array}$

$\beta$-Carotene (mcg per $1000 \mathrm{kcal})$

\begin{tabular}{lrrlll} 
Q1 & 615.9 & 292 & 317 & 1.00 & \\
Q2 & 986.0 & 273 & 316 & 1.04 & $(0.82,1.32)$ \\
Q3 & 1439.6 & 259 & 316 & 0.99 & $(0.77,1.26)$ \\
Q4 & 2347.6 & 244 & 317 & 0.99 & $(0.77,1.27)$ \\
$P_{\text {trend }}$ & & & & 0.838 & \\
\hline
\end{tabular}

Abbreviations: $\mathrm{Cl}=$ confidence intervals; $\mathrm{OR}=$ odds ratios. ${ }^{\mathrm{a}} \mathrm{P}_{\text {trend }}$ were calculated using the median value for each quartile. ${ }^{b}$ Adjusted for gender (female/male), age (0-54, 55-64, 65-74, 75 + years), region (Maine, Vermont, New Hampshire), race (White/other), Hispanic status (yes/no), smoking status (never, occasional, former, current), usual BMI ( $\mathrm{kgm}^{-2}-$ continuous), and total energy (kcal per day - continuous). 
García-Closas et al, 2007; Aune et al, 2009; Larsson et al, 2009; Ferrucci et al, 2010; Jakszyn et al, 2011). However, one cohort study found a positive association between total meat intake and bladder cancer (Mills et al, 1991), and another found an increased risk for high total processed meat intake ( $\mathrm{Hu}$ et al, 2011). Consumption of specific processed meat groups, such as bacon, cold cuts, ham, hot dogs, or sausages, have previously been linked to bladder cancer risk in some (Chyou et al, 1993; Michaud et al, 2006; Larsson et al, 2009; Ferrucci et al, 2010) but not all studies (Chyou et al, 1993; Michaud et al, 2006; Larsson et al, 2009; Ferrucci et al, 2010).

Meat is a source of multiple mutagenic compounds such as HCAs, PAHs, and $\mathrm{N}$-nitroso compounds (NOCs). Previous animal studies have demonstrated HCAs, such as MeIQx and PhIP, affects the urinary system as well as other organ systems (Ohgaki et al, 1987; Takahashi et al, 1993; Shirai et al, 1998). However, similar to our data, meat cooking preferences and mutagens have not been associated with bladder cancer in other studies (Augustsson et al, 1999; García-Closas et al, 2007; Ferrucci et al, 2010). Dietary intake of MeIQx, DiMeIQx, and PhIP in our study was lower than in a previous study in Spain (García-Closas et al, 2007), but was higher compared with another US study population (Ferrucci et al, 2010). Processed meat is also a source of NOCs, which are formed from nitrate and nitrite, and they can cause tumours at multiple organ sites in many animal species (Bogovski and Bogovski, 1981), including the urinary bladder (Mirvish, 1995). In a recent cohort study, processed meat intake was not associated with bladder cancer, but total nitrate and nitrite intake from processed meats had a borderline positive association with bladder cancer (RR: 1.29; 95\% CI: 1.00-1.67; $P=0.110$ ) (Ferrucci et al, 2010). In contrast, another recent cohort study that estimated NOC exposure in relation to bladder cancer was null (Jakszyn et al, 2011). Although the findings between HCAs, PAHs, and NOCs in relation to bladder cancer have been null or inconsistent, the weakness of effect may be due to the inherent design of epidemiological studies where exposures are measured from self-reported dietary intake of individual foods ensuing in non-differential misclassification of exposure and dilution of the observed effect (Eichholzer and Gutzwiller, 1998; Sinha, 2002).

In our study, we did not find any associations between fruit intake and bladder cancer. Other studies have also found null results (Shibata et al, 1992; Michaud et al, 1999; Nagano et al, 2000; Michaud et al, 2002); only one study has shown a statistically significant reduction in bladder cancer risk for individuals in the highest category of fruit intake compared with the lowest (Zeegers et al, 2001b). Other research studies have shown that specific fruit or vegetable groups have some protective effects but the findings are highly variable across the studies (Michaud et al, 1999; Nagano et al, 2000); for example, in a Spanish case-control study, berries were inversely related to bladder cancer, whereas in a Finnish cohort study there was no association (Michaud et al, 2002; García-Closas et al, 2007). We did not find any specific fruit or vegetable group to be protective against bladder cancer, which is similar to the findings of other prospective cohort studies (Shibata et al, 1992; Zeegers et al, 2001b; Michaud et al, 2002).

Two case-control and cohort studies have observed inverse associations with specific vitamins such as A, B12, C and E (Shibata et al, 1992; Bruemmer et al, 1996; Michaud et al, 2000; García-Closas et al, 2007); however, other studies have found no evidence for a protective effect of vitamins (Nomura et al, 1991; Riboli et al, 1991; Michaud et al, 1999; Wakai et al, 2000; Zeegers et al, 2001b; Michaud et al, 2002; Brinkman et al, 2010; Hotaling et al, 2011). Micronutrients can potentially prevent carcinogenesis and inhibit tumour growth through a multitude of biological mechanisms (Greenwald et al, 2001; Steinmetz and Potter, 1991); for example, carotenoids increase cell differentiation, vitamin $\mathrm{C}$ prevents formation of NOCs, vitamin $\mathrm{E}$ has intracellular antioxidant properties, and vitamin $\mathrm{D}$ and calcium decrease cellular proliferation (Steinmetz and Potter, 1991; Greenwald et al, 2001).

Dietary vitamin B12 was inversely associated with bladder cancer in our study; this association was also observed in a Spanish population in diet and vitamin supplements (García-Closas et al, 2007). The main sources of vitamin B12 include fish, meat, poultry, eggs, milk, and milk products, with $27 \%$ of the micronutrient coming from beef (Subar et al, 1998). Vitamin B12, as well as folic acid, is needed for methylation in DNA metabolism (Fenech, 2001 Fenech et al, 1998). Deficiencies in vitamin B12 may result in high incorporation of uracil into the DNA, thus leading to chromosomal breakage, which has implications for cancer risk. However, more studies are needed to elucidate the biological mechanism between vitamin B12 and bladder cancer specifically.

The strengths of this study are the large sample size and the comprehensive validated dietary questionnaire. Furthermore, this study was located in a region with high bladder cancer incidence and mortality (Brown et al, 1995). One of the limitations of our study is the $60 \%$ participation rate, although this was approximately the same among both the cases and controls. The main reason for non-participation in the cases was because $22 \%$ of the eligible cases had died before entry to the study. The main reason for non-participation among the controls was refusals, with $23.5 \%$ of eligible controls refusing to participate. Using data on some of the non-respondent cases from the state cancer registries, we observed that non-respondent cases did not seem to differ by sex or cancer stage compared with respondents. We also found a low percent of cancers were at high stage, and this was similar among those who participated and those who did not. Selection bias is a potential limitation in case-control studies; however, the characteristics of our study participants are similar to the NHANES 1999-2002 study population, which is representative of the general population in the USA (Ong et al, 2007; Seligman et al, 2007). In comparison with a cohort study, our study population characteristics, dietary intake and the associations observed was similar (Ferrucci et al, 2010). Moreover, the aggressive and non-aggressive bladder cancer cases in our study did not differ by major dietary factors (red and processed meat, fruits, and vegetables), age, race, Hispanic status, region, smoking, and BMI except for gender. As in most case-control studies of diet, there is also a concern regarding differential recall bias between cases and controls, which is a potential weakness of our study. Issues of measurement error associated with assessing usual dietary intake in large epidemiologic studies are also a concern but it will most likely be nondifferential misclassification resulting in an underestimation of the true association. Furthermore, since we investigated multiple exposures, there is a possibility that associations may have been observed by chance.

In summary, we observed a positive association between processed meat intake and bladder cancer, especially for processed red meat. Furthermore, individuals who consumed larger amounts of vitamin B12 had a decreased risk for this malignancy. Future studies are needed to clarify the role of processed meat and vitamin B12 in bladder carcinogenesis as we have seen some inconsistencies in the current literature.

\section{ACKNOWLEDGEMENTS}

We thank Anna McIntosh, Paul Hurwitz, Patricia Clark and Vanessa Olivo (Westat, Rockville, MD, USA) for their support in study and data management, and Anne Taylor and Mary McAdams (Information Management Services, Silver Spring, MD, USA) for their programming help. We would like to acknowledge Dr Michael Jones (Maine Medical Center), Sue Ledoux and Dawn Nicolaides (Maine Cancer Registry), Kimberley Walsh and Christina Robinson (Dartmouth Medical School), Dr Masatoshi 
Kida (University of Vermont), William Apao and Carolyn Greene (Vermont Cancer Registry) for their contributions during the fieldwork and data collection phases. We also thank all fieldwork staff, interviewers, and data abstractors for their dedicated work, and our study participants for agreeing to be part of this study. This research was supported by the Intramural Research Program

\section{REFERENCES}

Alaejos MS, González V, Afonso AM (2008) Exposure to heterocyclic aromaticof cooked red meat and its effect on human cancer risk: A review. Food Contam 25(1): 2-24

Asaduzzaman Khan M, Tania M, Zhang D, Chen H (2010) Antioxidant enzymes and cancer. Chin J Cancer Res 22(2): 87-92

Augustsson K, Skog K, Jagerstad M, Dickman PW, Steineck G (1999) Dietary heterocyclic amines and cancer of the colon, rectum, bladder, and kidney: a population-based study. Lancet 353(9154): 703-707

Aune D, De Stefani E, Ronco A, Boffetta P, Deneo-Pellegrini H, Acosta G, Mendilaharsu M (2009) Meat consumption and cancer risk: a casecontrol study in Uruguay. Asian Pac J Cancer Prev 10(3): 429-436

Balbi J, Larrinaga M, De Stefani E, Mendilaharsu M, Ronco A, Boffetta P, Brennan P (2001) Foods and risk of bladder cancer: a case-control study in Uruguay. Eur J Cancer Prev 10(5): 453

Baris D, Karagas MR, Verrill C, Johnson A, Andrew AS, Marsit CJ, Schwenn M, Colt JS, Cherala S, Samanic C (2009) A case-control study of smoking and bladder cancer risk: emergent patterns over time. J Natl Cancer Inst 101(22): 1553-1561

Bogovski P, Bogovski S (1981) Special report animal species in which $n$-nitroso compounds induce cancer. Int J Cancer 27(4): 471-474

Brinkman MT, Karagas MR, Zens MS, Schned A, Reulen RC, Zeegers MP (2010) Minerals and vitamins and the risk of bladder cancer: results from the New Hampshire Study. Cancer Causes Control 21(4): 609-619

Brown LM, Zahm SH, Hoover RN, Fraumeni JF (1995) High bladder cancer mortality in rural New England (United States): an etiologic study. Cancer Causes Control 6(4): 361-368

Bruemmer B, White E, Vaughan T, Cheney C (1996) Nutrient intake in relation to bladder cancer among middle-aged men and women. $A m J$ Epidemiol 144(5): 485

Chyou P, Nomura A, Stemmermann G (1993) A prospective study of diet, smoking, and lower urinary tract cancer. Ann Epidemiol 3(3): 211-216

Cross A, Leitzmann M, Gail M, Hollenbeck A, Schatzkin A, Sinha R (2007) A prospective study of red and processed meat intake in relation to cancer risk. PLoS Med 4(12): e325

Dixon L, Zimmerman T, Kahle L, Subar A (2003) Adding carotenoids to the NCI Diet History Questionnaire database. J Food Composition Anal 16(3): 269-280

Eichholzer M, Gutzwiller F (1998) Dietary nitrates, nitrites, and $N$-nitroso compounds and cancer risk: a review of the epidemiologic evidence. Nutr Rev 56(4): 95-105

Fenech M (2001) The role of folic acid and Vitamin B12 in genomic stability of human cells. Mutat Res 475(1-2): 57-67

Fenech M, Aitken C, Rinaldi J (1998) Folate, vitamin B12, homocysteine status and DNA damage in young Australian adults. Carcinogenesis 19(7): 1163

Ferrucci LM, Sinha R, Ward MH, Graubard BI, Hollenbeck AR, Kilfoy BA, Schatzkin A, Michaud DS, Cross AJ (2010) Meat and components of meat and the risk of bladder cancer in the NIH-AARP Diet and Health Study. Cancer 116(18): 4345-4353

García-Closas R, García-Closas M, Kogevinas M, Malats N, Silverman D, Serra C, Tardón A, Carrato A, Castaño-Vinyals G, Dosemeci M (2007) Food, nutrient and heterocyclic amine intake and the risk of bladder cancer. Eur J Cancer 43(11): 1731-1740

Greenwald P, Clifford C, Milner J (2001) Diet and cancer prevention. Eur J Cancer 37(8): 948

Hashida C, Nagayama K, Takemura N (1982) Induction of bladder cancer in mice by implanting pellets containing tryptophan pyrolysis products. Cancer Lett 17(1): 101-105

Hotaling JM, Wright JL, Pocobelli G, Bhatti P, Porter MP, White E (2011) Long-term use of supplemental vitamins and minerals does not reduce the risk of urothelial cell carcinoma of the bladder in the VITamins And Lifestyle Study. J Urol 185(4): 1210-1215

Howlader N, Noone A, Krapcho M, Neyman N, Aminou R, Waldron W, Altekruse S, Kosary C, Ruhl J, Tatalovich Z, Cho H, Mariotto A, Eisner M, of the National Cancer Institute, National Institutes of Health, Department of Health and Human Services. The authors have nothing to disclose.

Supplementary Information accompanies the paper on British Journal of Cancer website (http://www.nature.com/bjc)

Lewis D, Chen H, Feuer E, Cronin K, Edwards B (2010) SEER Stat Fact Sheets: Bladder. http://seer.cancer.gov/csr/1975_2008/. Last accessed 19 March 2012

Hu J, La Vecchia C, Morrison H, Negri E, Mery L (2011) Salt, processed meat and the risk of cancer. Eur J Cancer Prev 20(2): 132

Jakszyn P, González CA, Luján-Barroso L, Ros MM, Bueno-de-Mesquita HB, Roswall N, Tjønneland AM, Büchner FL, Egevad L, Overvad K (2011) Red Meat, Dietary Nitrosamines, and Heme Iron and Risk of Bladder Cancer in the European Prospective Investigation into Cancer and Nutrition (EPIC). Cancer Epidemiol Biomarkers Prev 20(3): 555

Jiang Z, Li C, Wang X (2011) Glutathione S-transferase M1 polymorphism and bladder cancer risk: a meta-analysis involving 33 studies. Exp Biol Med 236(6): 723-728

Lampe JW, King IB, Li S, Grate MT, Barale KV, Chen C, Feng Z, Potter JD (2000) Brassica vegetables increase and apiaceous vegetables decrease cytochrome P450 1A2 activity in humans: changes in caffeine metabolite ratios in response to controlled vegetable diets. Carcinogenesis 21(6): 1157

Larsson S, Johansson J, Andersson S, Wolk A (2009) Meat intake and bladder cancer risk in a Swedish prospective cohort. Cancer Causes Control 20(1): 35-40

Leppert JT, Shvarts O, Kawaoka K, Lieberman R, Belldegrun AS, Pantuck AJ (2006) Prevention of bladder cancer: a review. European Urology 49(2): 226-234

Marmot M, Atinmo T, Byers T, Chen J, Hirohata T, Jackson A, James W, Kolonel L, Kumanyika S, Leitzmann C (2007) Food, nutrition, physical activity, and the prevention of cancer: a global perspective. World Cancer Res Fund/Am Inst Cancer 46(4): 312-314

Michaud D, Holick C, Giovannucci E, Stampfer M (2006) Meat intake and bladder cancer risk in 2 prospective cohort studies. Am J Clin Nutr 84(5): 1177

Michaud D, Pietinen P, Taylor P, Virtanen M, Virtamo J, Albanes D (2002) Intakes of fruits and vegetables, carotenoids and vitamins $\mathrm{A}, \mathrm{E}, \mathrm{C}$ in relation to the risk of bladder cancer in the ATBC cohort study. $\mathrm{Br} J$ Cancer 87(9): 960-965

Michaud D, Spiegelman D, Clinton S, Rimm E, Willett W, Giovannucci E (2000) Prospective study of dietary supplements, macronutrients, micronutrients, and risk of bladder cancer in US men. Am J Epidemiol 152(12): 1145

Michaud DS, Spiegelman D, Clinton SK, Rimm EB, Willett WC, Giovannucci EL (1999) Fruit and vegetable intake and incidence of bladder cancer in a male prospective cohort. JNCI J Natl Cancer Inst 91(7): 605

Mills P, Beeson W, Phillips R, Fraser G (1991) Bladder cancer in a low risk population: results from the Adventist Health Study. Am J Epidemiol 133(3): 230

Mirvish SS (1995) Role of $N$-nitroso compounds (NOC) and $N$-nitrosation in etiology of gastric, esophageal, nasopharyngeal and bladder cancer and contribution to cancer of known exposures to NOC. Cancer Lett 93(1): 17-48

Nagano J, Kono S, Preston DL, Moriwaki H, Sharp GB, Koyama K, Mabuchi $\mathrm{K}$ (2000) Bladder-cancer incidence in relation to vegetable and fruit consumption: a prospective study of atomic-bomb survivors. Int $J$ Cancer 86(1): 132-138

Nomura AMY, Kolonel LN, Hankin JH, Yoshizawa CN (1991) Dietary factors in cancer of the lower urinary tract. Int $J$ Cancer 48(2): 199-205

Ohgaki H, Hasegawa H, Suenaga M, Sato S, Takayama S, Sugimura T (1987) Carcinogenicity in mice of a mutagenic compound, 2-amino-3, 8-dimethylimidazo [4, 5-f] quinoxaline (MeIQx) from cooked foods. Carcinogenesis 8(5): 665

Ong KL, Cheung BM, Man YB, Lau CP, Lam KS (2007) Prevalence, awareness, treatment, and control of hypertension among United States adults 1999-2004. Hypertension 49(1): 69-75 
Pelucchi C, Bosetti C, Negri E, Malvezzi M, La Vecchia C (2006) Mechanisms of disease: the epidemiology of bladder cancer. Nat Clin Pract Urol 3(6): 327-340

Riboli E, González CA, López Abente G, Errezola M, Izarzugaza I, Escolar A, Nebot M, Hémon B, Agudo A (1991) Diet and bladder cancer in Spain: a multi centre case control study. Int J Cancer 49(2): 214-219

Seligman HK, Bindman AB, Vittinghoff E, Kanaya AM, Kushel MB (2007) Food insecurity is associated with diabetes mellitus: results from the National Health Examination and Nutrition Examination Survey (NHANES) 1999-2002. J Gen Intern Med 22(7): 1018-1023

Shibata A, Paganini-Hill A, Ross R, Henderson B (1992) Intake of vegetables, fruits, beta-carotene, vitamin $\mathrm{C}$ and vitamin supplements and cancer incidence among the elderly: a prospective study. $\mathrm{Br} \mathrm{J}$ Cancer 66(4): 673

Shirai T, Takahashi S, Cui L, Yamada Y, Tada M, Kadlubar FF, Ito N (1998) Use of polyclonal antibodies against carcinogen-DNA adducts in analysis of carcinogenesis. Toxicol Lett 102: 441-446

Sinha R (2002) An epidemiologic approach to studying heterocyclic amines. Mutat Res 506-507: 197-204

Sinha R, Cross A, Curtin J, Zimmerman T, McNutt S, Risch A, Holden J (2005) Development of a food frequency questionnaire module and databases for compounds in cooked and processed meats. Mol Nutr Food Res 49(7): 648-655

Steineck G, Hagman U, Gerhardsson M, Norell SE (1990) Vitamin A supplements, fried foods, fat and urothelial cancer. A case-referent study in Stockholm in 1985-87. Int J Cancer 45(6): 1006-1011

Steinmetz K, Potter J (1991) Vegetables, fruit, and cancer. II. Mechanisms. Cancer Causes Control 2(6): 427-442

Subar AF, Krebs-Smith SM, Cook A, Kahle LL (1998) Dietary sources of nutrients among US adults, 1989 to 1991. J Am Diet Ass 98(5): 537-547
Subar AF, Thompson FE, Kipnis V, Midthune D, Hurwitz P, McNutt S, McIntosh A, Rosenfeld S (2001) Comparative validation of the Block, Willett, and National Cancer Institute food frequency questionnaires: the Eating at America's Table Study. Am J Epidemiol 154(12): 1089-1099

Takahashi M, Toyoda K, Aze Y, Furuta K, Mitsumori K, Hayashi Y (1993) The rat urinary bladder as a new target of heterocyclic amine carcinogenicity: tumor induction by 3 -amino-1-methyl-5H-pyrido [4, 3-b] indole acetate. Jpn J Cancer Res 84(8): 852-858

Tavani A, La Vecchia C, Gallus S, Lagiou P, Trichopoulos D, Levi F, Negri E (2000) Red meat intake and cancer risk: a study in Italy. Int J Cancer 86(3): 425-428

Thompson F, Kipnis V, Midthune D, Freedman L, Carroll R, Subar A, Brown C, Butcher M, Mouw T, Leitzmann M (2008) Performance of a food-frequency questionnaire in the US NIH-AARP (National Institutes of Health-American Association of Retired Persons) Diet and Health Study. Public Health Nutr 11(02): 183-195

Wakai K, Takashi M, Okamura K, Yuba H, Suzuki K, Murase T, Obata K, Itoh H, Kato T, Kobayashi M (2000) Foods and nutrients in relation to bladder cancer risk: a case-control study in Aichi Prefecture, central Japan. Nutr Cancer 38(1): 13-22

Zeegers M, Goldbohm R, Van den Brandt P (2001a) Are retinol, vitamin C, vitamin $\mathrm{E}$, folate and carotenoids intake associated with bladder cancer risk? Results from the Netherlands Cohort Study. Br J Cancer 85(7): 977

Zeegers M, Goldbohm R, van den Brandt P (2001b) Consumption of Vegetables and Fruits and Urothelial Cancer Incidence. Cancer Epidemiol Biomarkers Prev 10(11): 1121

Zheng W, Lee SA (2009) Well-done meat intake, heterocyclic amine exposure, and cancer risk. Nutr Cancer 61(4): 437-446

This work is published under the standard license to publish agreement. After 12 months the work will become freely available and the license terms will switch to a Creative Commons Attribution-NonCommercial-Share Alike 3.0 Unported License. 